\title{
Quality of life in patients with intracranial tumors: does tumor laterality matter?
}

\author{
Christina Drewes, MD, ${ }^{1}$ Lisa Millgård Sagberg, MSc, ${ }^{2,3}$ Asgeir Store Jakola, PhD, ${ }^{2,4,5}$ and \\ Ole Solheim, $\mathrm{PhD}^{2,3}$
}

\begin{abstract}
Departments of ${ }^{1}$ Anesthesiology and ${ }^{2}$ Neurosurgery, St. Olavs University Hospital, Trondheim; ${ }^{3}$ Department of Neuroscience Norwegian University of Science and Technology, Trondheim, Norway; ${ }^{4}$ Department of Neurosurgery, Gothenburg University Hospital, Gothenburg; and Institute of Neuroscience and Physiology, University of Gothenburg, Sahlgrenska Academy, Gothenburg, Sweden
\end{abstract}

OBJECTIVE Traditionally, the dominant (usually left) cerebral hemisphere is regarded as the more important one, and everyday clinical decisions are influenced by this view. However, reported results on the impact of lesion laterality are inconsistent in the scarce literature on quality of life $(\mathrm{QOL})$ in patients with brain tumors. The authors aimed to study which cerebral hemisphere is the most important to patients with intracranial tumors with respect to health-related QOL (HRQOL).

METHODS Two hundred forty-eight patients with unilateral, unifocal gliomas or meningiomas scheduled for primary surgery were included in this prospective cohort study. Generic HRQOL was measured using the EQ-5D-3L questionnaire preoperatively and after 4-6 weeks. Cross-sectional and longitudinal analyses of data were performed.

RESULTS Tumor volumes were significantly larger in right-sided tumors at diagnosis, and language or speech problems were more common in left-sided lesions. Otherwise, no differences existed in baseline data. The median EQ-5D-3L index was 0.73 (range -0.24 to 1.00) in patients with right-sided tumors and 0.76 (range -0.48 to 1.00 ) in patients with left-sided tumors $(p=0.709)$. Due to the difference in tumor volumes at baseline, histopathology and tumor volumes were matched in 198 patients. EQ-5D-3L index scores in this $1: 1$ matched analysis were 0.74 (range -0.7 to 1.00 ) for patients with right-sided and 0.76 (range -0.48 to 1.00) for left-sided lesions $(p=0.342$ ). In the analysis of longitudinal data, no association was found between tumor laterality and postoperative EQ-5D-3L index scores $(p=0.957)$ or clinically significant change in HRQOL following surgery $(p=0.793)$.

CONCLUSIONS In an overall patient-reported QOL perspective, tumor laterality does not appear to be of significant importance for generic HRQOL in patients with intracranial tumors. This may imply that right-sided cerebral functions are underestimated by clinicians.

http://thejns.org/doi/abs/10.3171/2015.12.JNS152252

KEY WORDS brain neoplasm; laterality; quality of life; oncology

$\mathrm{B}$ Y tradition, neurosurgeons treat the left cerebral hemisphere with particular respect. The special consideration for the left hemisphere is mainly based on the fact that it is the dominant one for eloquent functions in $90 \%-96 \%$ of right-handed as well as in $70 \%-86 \%$ of left-handed individuals..$^{12,20}$ Because $90 \%$ of people are right-handed,, 314 the vast majority of patients rely on their left hemisphere for important functions, such as speech and fine motor skills performed by the dominant hand. Various clinical decisions are influenced by the greater respect for the left hemisphere. This includes planning of surgical approaches to midline pathology, endoscopic approaches to the ventricle system, and placement of ventricle drains or intracranial pressure monitoring devices. Laterality of a lesion may also be a factor when deciding for or against evacuation of traumatic or spontaneous hematomas, or when decompressive hemicraniectomy for severe traumatic brain injury or malignant stroke is considered. Moreover, laterality is often taken into account when surgical strategies for brain tumors are planned. This

ABBREVIATIONS ASA = American Society of Anesthesiologists; $\mathrm{HRQOL}=$ health-related QOL; $K P S=$ Karnofsky Performance Scale; $\mathrm{MCID}=$ minimum clinically important difference; $\mathrm{QOL}=$ quality of life; VAS = visual analog scale.

SUBMITTED September 25, 2015. ACCEPTED December 11, 2015.

INCLUDE WHEN CITING Published online March 25, 2016; DOI: 10.3171/2015.12.JNS152252. 
includes decisions on the use of surgical tools or anesthetic techniques, such as awake craniotomy, intraoperative mapping, or monitoring techniques; decisions on how much tissue to remove safely; and sometimes, the decision on whether to operate at all.

However, is the right cerebral hemisphere really less important to our patients than the left, "dominant" hemisphere? Two studies on ischemic stroke report no difference in functional outcome based on stroke laterality. ${ }^{6,7}$ Furthermore, a recent case series on patients after decompressive hemicraniectomy for ischemic stroke found no difference in health-related quality of life (HRQOL) between patients with left versus those with right hemisphere infarctions. ${ }^{23}$ In a study of patients with brain tumors, tumor laterality could neither predict survival nor postoperative Karnofsky Performance Scale (KPS) score.${ }^{16}$ However, the literature is scarce when it comes to the influence of brain tumor laterality on HRQOL. Two studies from the same cohort found that right-sided tumors were associated with a poorer HRQOL than left-sided tumors were and, more specifically, that there was a higher incidence of anxiety in patients with right-sided tumors. ${ }^{11,19} \mathrm{~A}$ few other, highly heterogeneous studies report HRQOL and neuropsychological outcomes related to tumor laterality as minor parts of their findings., ${ }^{9,10,17,24}$

In recent years, advanced methods (such as functional MRI, intraoperative imaging, and awake surgery) have facilitated a distinction between eloquent and noneloquent brain regions, and thereby individualized tailoring of treatment strategies. Yet laterality continues to be part of everyday clinical decisions. More research on the influence of lesion laterality on patient-reported outcomes is needed. In this prospective cohort study in patients with intracranial tumors, we aimed to explore whether patientreported HRQOL before and after surgery is dependent on tumor laterality.

\section{Methods}

Adults (18 years or older) undergoing first-time surgery for gliomas and meningiomas at the Department of Neurosurgery at St. Olavs University Hospital (Trondheim, Norway) between January 2007 and March 2015 were found eligible for inclusion after providing written informed consent. All tumors were histopathologically confirmed. Our tertiary institution serves a geographically defined catchment area, ensuring population-based, unselected referral. Only unifocal tumors with supratentorial and unilateral location in either the left or the right cerebral hemisphere were included. Patients with lesions involving the midline, brainstem, or cerebellum were excluded. Noninclusion because of lack of consent was mainly due to administrative/staffing issues. For the inclusion process, please see Fig. 1.

\section{Data Collection}

Patient-reported QOL using the EQ-5D-3L questionnaire was recorded 1-3 days before surgery and at 4-6 weeks (median 36 days, range 23-131 days) postoperatively, the latter in a structured telephone interview, performed by a research nurse. During the first years of our data collection, EQ-5D visual analog scale (VAS) scores were not collected, and we only had VAS data on 123 of 248 patients. KPS score was also recorded prospectively. Other clinical variables, imaging findings, and histopathological variables were obtained retrospectively from hospital records, operative reports, and pathology reports.

\section{The EQ-5D-3L Questionnaire}

The EQ-5D-3L is a generic QOL instrument that covers the 5 dimensions of mobility, self-care, usual activities, pain/discomfort, and anxiety/depression. For each domain, the respondent can apply 1 of 3 grades: "no problems," "some problems," or "extreme problems." A single summary index value is calculated from the responses to the subdomains, ranging from "1" ("perfect health") to "0" ("equal to death"). Negative index values are possible, indicating a QOL worse than death. The EQ-5D-3L has been validated for the Norwegian population. ${ }^{13}$

The minimum clinically important difference (MCID) represents "the smallest difference in score in the domain of interest that patients perceive as important, either beneficial or harmful, and which would lead the clinician to consider a change in the patient's management." ${ }^{\text {MCID }}$ in patients undergoing glioma surgery has been found to range from 0.13 to $0.15,{ }^{18}$ which is slightly higher than in some other studied conditions. ${ }^{15}$

\section{Assessment of Images}

The patients underwent 1.5- or 3.0-T MRI at 1-33 days before surgery and within 72 hours postoperatively. The interpretation of image data was performed by 1 of 2 of the coauthors (O.S. or A.S.J.). The volumes of sphericalshaped lesions were calculated by using the ellipsoid volume formula $(4 / 3 \cdot \pi \mathrm{r} 1 \mathrm{r} 2 \mathrm{r} 3)$ based on the maximum tumor diameters in the available image dimensions. In cup-shaped residual tumors, volume was calculated by subtracting the ellipsoid-shaped resection cavity from the volume of the tumor/cavity complex, as performed earlier by others. ${ }^{22}$ In very complex configurations, we defined several volumes within the residual lesion and measured using the above-mentioned methods as appropriate, then summed up these volumes.

\section{Ethics}

Data collection was approved by the Committee for Medical and Health Research Ethics for Health Region Mid-Norway and adhered to the guidelines of the Helsinki Declaration. All included patients provided their explicit written, informed consent.

\section{Statistical Analyses \\ Power Analysis}

Values for standard deviation (0.26) and MCID (0.14) were obtained from published literature about gliomas. ${ }^{18}$ Using sample power, we found that 55 patients in each group were necessary to provide $80 \%$ power. Anticipating the possibility for skewed distribution of our primary outcome (EQ-5D-3L index), we added $15 \%$ to this patient number, resulting in 63 patients per group.

All statistical analyses were performed using SPSS 


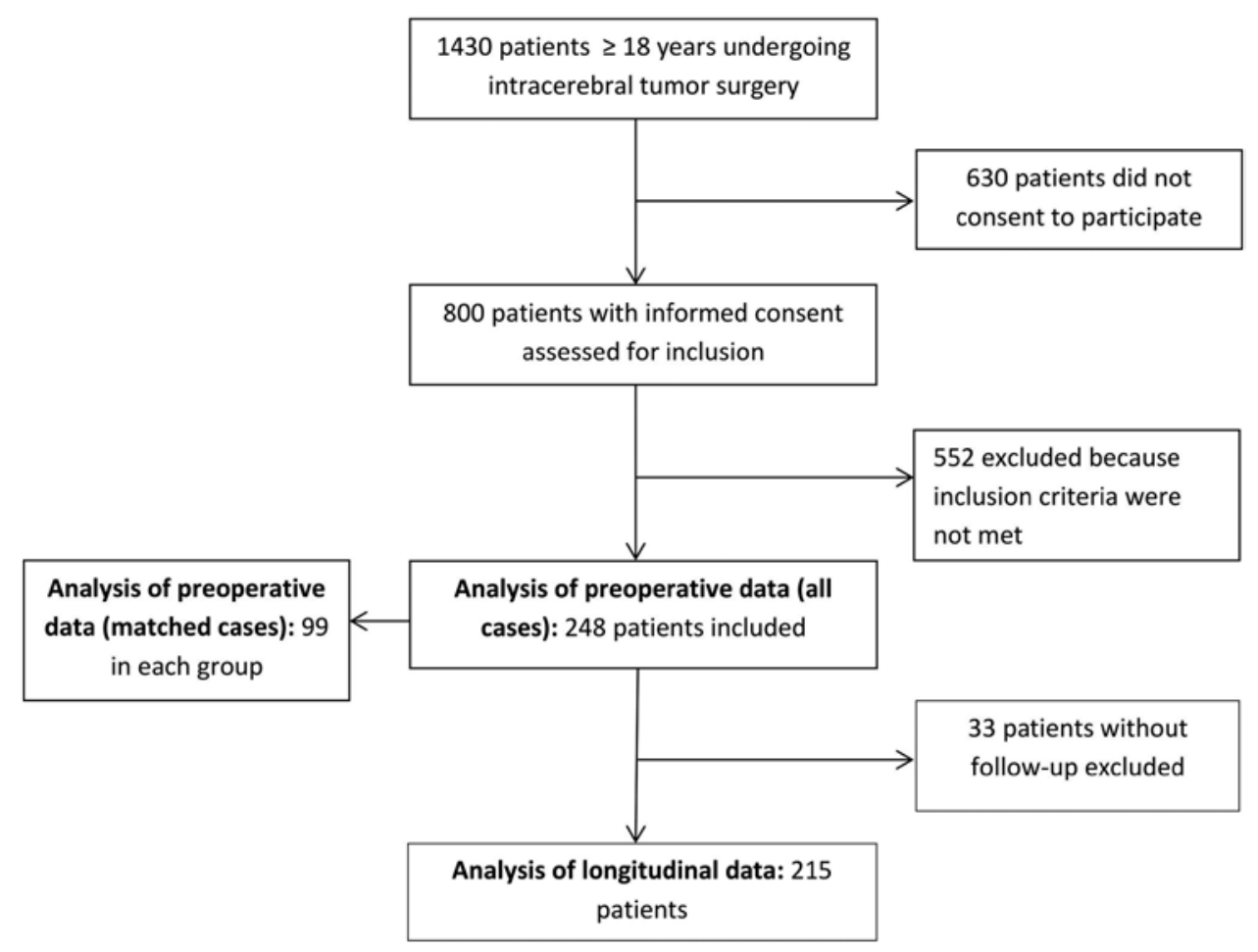

FIG. 1. Flow chart of the inclusion process.

(version 21.0). The statistical significance level was set to 2-sided ( $\mathrm{p}<0.05)$. Q-Q plots were used to test for normal distribution of data. Two-sided t-tests were used for comparison of means in normally distributed data. The Mann-Whitney U-test was used as the nonparametric test for ranked comparison of medians in non-normally distributed data. The chi-square test (with continuity correction in $2 \times 2$ contingency tables) was used for comparisons of categorical variables. The Fisher's exact test was used for the expected number $\leq 5$ per cell. Spearman's rho was used for correlation analyses in skewed data.

\section{Missing Data}

Thirty-three patients without HRQOL data at followup were excluded from the longitudinal analysis. Incomplete data for 10 of 1240 single EQ-5D-3L subdomains in 8 different patients $(0.8 \%)$ in the preoperative analysis and incomplete data for 2 single EQ-5D-3L subdomains in 2 different patients $(0.2 \%)$ in the longitudinal analysis were imputed by sample mean. Postoperative tumor volume was missing in 4 cases due to lack of postoperative MR images because of medical (pacemaker, renal failure) or administrative (transfer to other hospital) reasons. Time to follow-up was missing due to administrative reasons in 4 cases in the longitudinal group. These values were handled as "missing" by pairwise exclusion in SPSS.

\section{Results}

\section{Analysis of Preoperative Data}

As seen in Table 1, most preoperative baseline variables from the 248 included patients were evenly distributed between patients with right- and left-sided tumors.
However, preoperative tumor volumes were significantly larger in patients with right-sided than in patients with left-sided tumors $(\mathrm{p}=0.019)$. As expected, language problems occurred significantly more often in patients with left-sided tumors $(\mathrm{p}<0.001)$. Preoperative KPS scores were comparable between the laterality groups. There was no difference in median preoperative EQ-5D-3L indexes in patients with right-sided versus those with left-sided tumors $(\mathrm{p}=0.709)$. Figure 2 depicts the percentage of patients with right- versus left-sided tumors who reported any problems on the EQ-5D-3L questionnaire. As noted, results were not significantly affected by tumor laterality.

EQ-5D VAS data were available for only 123 patients (63 with right-sided and 60 with left-sided tumors). An analysis of these data did not show any statistically significant difference in VAS scores between the laterality groups (mean 65.4 in right-sided lesions and mean 68.0 in left-sided lesions; $\mathrm{p}=0.537$ ).

Because the difference in tumor volumes at baseline could act as a possible confounding factor, we decided to match cases for tumor volumes within histopathological entities. For the matching procedure, tumor volumes in right- and left-sided tumors were grouped into quartiles. We then performed a pairwise matching (right-left) of tumor volumes of similar size (same volume quartile) and same histopathology (low-grade glioma, high-grade glioma, meningioma), resulting in comparable average tumor volumes within quartiles. Cases with no match in histopathology and volume (within the same quartile) were excluded from the 1:1 matched analyses. The matching procedure was performed blinded for QOL scores and all clinical variables other than the matching variables (tumor volume and histopathology). As is shown in Table 1 and 
TABLE 1. Baseline data and preoperative EQ-5D-3L index values in all patients and the matched cohort*

\begin{tabular}{|c|c|c|c|c|c|c|}
\hline \multirow[b]{2}{*}{ Variable } & \multicolumn{3}{|c|}{ All Patients $(n=248)$} & \multicolumn{3}{|c|}{ Matched Cohort $(n=198)$} \\
\hline & $\begin{array}{l}\text { Rt-Sided Tumor } \\
\quad(n=119)\end{array}$ & $\begin{array}{l}\text { Lt-Sided Tumor } \\
\quad(n=129)\end{array}$ & p Value & $\begin{array}{l}\text { Rt-Sided Tumor } \\
\quad(\mathrm{n}=99)\end{array}$ & $\begin{array}{l}\text { Lt-Sided Tumor } \\
\qquad(\mathrm{n}=99)\end{array}$ & $p$ Value \\
\hline Females (\%) & $58(48.7)$ & $61(47.3)$ & 0.919 & $50(50.5)$ & $46(46.5)$ & 0.670 \\
\hline Mean age \pm SD (yrs) & $56.7 \pm 14.0$ & $57.2 \pm 15.4$ & 0.800 & $57.4 \pm 13.6$ & $56.7 \pm 15.6$ & 0.727 \\
\hline ASA class $(\%)$ & & & 1.000 & & & 0.868 \\
\hline $1-2$ & $90(75.6)$ & $97(75.2)$ & & $74(74.7)$ & $76(76.8)$ & \\
\hline $3-4$ & $29(24.4)$ & $32(24.8)$ & & $25(25.3)$ & $23(23.2)$ & \\
\hline \multicolumn{7}{|l|}{ Preop symptoms (\%) } \\
\hline Headache & $58(48.7)$ & $40(31)$ & 0.06 & $46(46.5)$ & $32(32.3)$ & 0.059 \\
\hline Seizures & $44(37)$ & $41(31.8)$ & 0.467 & $38(38.4)$ & $31(31.3)$ & 0.371 \\
\hline Cognitive & $28(23.5)$ & $38(29.5)$ & 0.362 & $23(23.2)$ & $32(32.3)$ & 0.204 \\
\hline Nausea/vomiting & $13(10.9)$ & $5(3.9)$ & 0.058 & $11(11.1)$ & $3(3)$ & 0.052 \\
\hline Balance/coordination & $35(29.4)$ & $30(23.3)$ & 0.339 & $26(26.3)$ & $23(23.2)$ & 0.742 \\
\hline Visual disturbances & $10(8.4)^{\prime}$ & $14(10.9)$ & 0.662 & $9(9.1)$ & $12(12.1)$ & 0.644 \\
\hline Language/speech & $7(5.9)$ & $39(30.2)$ & $<0.001$ & $7(7.1)$ & $26(26.3)$ & 0.001 \\
\hline Cranial nerve symptoms & $15(12.6)$ & $12(9.3)$ & 0.529 & $13(13.1)$ & $8(8.1)$ & 0.356 \\
\hline Motor deficits & $26(21.9)$ & $19(14.7)$ & 0.198 & $20(20.2)$ & $17(17.2)$ & 0.715 \\
\hline KPS score $(\%)$ & & & 0.539 & & & 0.886 \\
\hline 100 & $27(22.7)$ & $23(17.8)$ & & $20(20.2)$ & $17(17.2)$ & \\
\hline 90 & $40(33.6)$ & $48(37.2)$ & & $37(37.4)$ & $38(38.4)$ & \\
\hline 80 & $25(21)$ & $35(27.1)$ & & $21(21.2)$ & $24(24.2)$ & \\
\hline 70 & $12(10.1)$ & $8(6.2)$ & & $10(10.1)$ & $7(7.1)$ & \\
\hline$\leq 60$ & $15(12.6)$ & $15(11.6)$ & & $11(11.1)$ & 13 (13.1) & \\
\hline Histopathology (\%)† & & & 0.320 & & & 1.000 \\
\hline High-grade glioma & $51(42.9)$ & $63(48.8)$ & & $15(15.2)$ & $15(15.2)$ & \\
\hline Low-grade glioma & $25(21.0)$ & $18(14.0)$ & & $44(44.4)$ & $44(44.4)$ & \\
\hline Meningioma & $43(36.1)$ & $48(37.2)$ & & $40(40.4)$ & $40(40.4)$ & \\
\hline Median tumor vol in $\mathrm{cm}^{3}$ (range) $\ddagger$ & $24.7(1.3-159.0)$ & $18.9(0.7-160.4)$ & 0.019 & $21.9(1.32-153.47)$ & $22.3(0.71-160.37)$ & 0.932 \\
\hline \multicolumn{7}{|l|}{ Localization $(\%) \S$} \\
\hline Frontal & $60(50.4)$ & $72(55.8)$ & 0.470 & $47(47.5)$ & $58(58.6)$ & 0.154 \\
\hline Temporal & $49(41.2)$ & $58(45.0)$ & 0.636 & $43(43.4)$ & $45(45.5)$ & 0.886 \\
\hline Parietal & $28(23.5)$ & $27(20.9)$ & 0.734 & $25(25.3)$ & $20(20.2)$ & 0.498 \\
\hline Occipital & $10(8.4)$ & $5(3.9)$ & 0.220 & $8(8.1)$ & $3(3.0)$ & 0.215 \\
\hline Unilateral deep $\mathbb{Z}$ & $18(15.1)$ & $13(10.1)$ & 0.313 & $10(10.1)$ & $11(11.1)$ & 1.000 \\
\hline Lobes involved (\%) & & & 0.582 & & & 0.840 \\
\hline 1 & $77(64.7)$ & $89(69.0)$ & & $68(68.7)$ & $66(66.7)$ & \\
\hline 2 & $38(31.9)$ & $34(26.4)$ & & $28(28.3)$ & $28(28.3)$ & \\
\hline 3 & $4(3.4)$ & $6(4.7)$ & & $3(3.0)$ & $5(5.1)$ & \\
\hline Median EQ-5D-3L index (range) & $0.73(-0.24$ to 1.00$)$ & $0.76(-0.48$ to 1.00$)$ & 0.709 & $0.74(-0.7$ to 1.00$)$ & $0.76(-0.48$ to 1.00$)$ & 0.342 \\
\hline $\begin{array}{l}\text { * Boldface type indicates statistical sig } \\
\text { † Confirmed by neuropathologist. } \\
\text { † Assessed from preoperative MR ima } \\
\S \text { More than } 1 \text { lobe may be affected. } \\
\text { T Basal ganglia, thalamus. }\end{array}$ & & & & & & \\
\hline
\end{tabular}

Fig. 3, results for the matched cohort were also not significantly affected by the sidedness of pathology.

\section{Analysis of Postoperative Data}

Longitudinal HRQOL data were available in 215 (87\%) of the 248 patients. Except for preoperative tumor volume, patient characteristics in the longitudinal analysis were evenly distributed between patients with right-sided versus those with left-sided tumors (Table 2). This also includes extent of resection and postoperative tumor volumes. The median postoperative EQ-5D-3L index 4-6 weeks after surgery was $0.80(\mathrm{p}=0.957)$ for both patients with rightsided and those with left-sided tumors. We calculated the change from preoperative to postoperative EQ-5D-3L index scores with a MCID of 0.14 as the cutoff value. There was no statistically significant difference between the 2 laterality groups when comparing percentages of patients whose EQ-5D-3L index values had improved, were unchanged, or had become worse.

In the longitudinal analysis of data, we considered developing a regression model to adjust for preoperative tumor volume, the only assessed factor with a statistically 


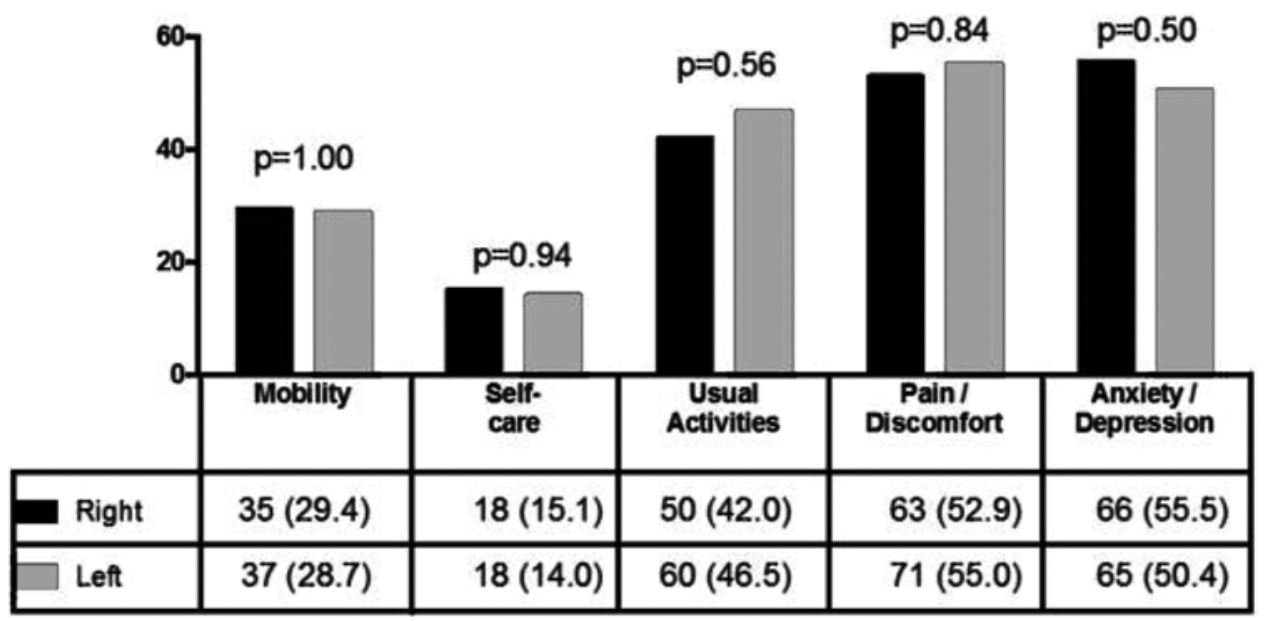

FIG. 2. Percentage of respondents with left- vs right-sided tumor reporting any problem on the EQ-5D-3L questionnaire at baseline.

significant difference between cohorts (Table 2). However, there was no correlation between preoperative tumor volume and EQ-5D-3L index at baseline (Spearman's rho = $-0.075, \mathrm{p}=0.239)$ and no correlation between preoperative tumor volume and EQ-5D-3L at follow-up (Spearman's rho $=-0.032, p=0.641$ ). Thus, preoperative tumor volume is not likely to be a confounding factor, and assumptions for a good regression model were not met.

\section{Discussion}

In this adequately powered prospective observational study, we found that patient-reported overall QOL, measured with the EQ-5D-3L, was neither clinically nor statistically different in patients with tumors in the left versus the right cerebral hemisphere. Our findings indicate that, at the group level, tumor laterality is of little importance for overall QOL. This applies to both preoperative and postoperative HRQOL data. Interestingly, preoperative tumor volumes were significantly larger in patients with right-sided tumors, possibly reflecting the fact that speech problems and fine motor deficits of the dominant hand are brought to medical attention sooner than deficits associated with right-sided lesions. However, matching of cases for tumor volumes within the histopathological subgroups did not alter our findings.

Measuring specific neurological deficits would undoubtedly have yielded different results. Language problems, for instance, are by far most common in left-sided lesions and left-sided surgery, as confirmed by our analysis of preoperative symptoms. For clinicians, language and motor deficits are often easier to detect and receive more focus than cognitive or affective problems. Nevertheless, functions located in the right hemisphere may still be of comparable importance to patients when it comes to overall QOL. The EQ-5D-3L, the instrument used in this study, includes the dimensions mobility, self-care, usual activities, pain/discomfort, and anxiety/depression. Our findings suggest that there may be consequences for right-hemisphere lesions that affect the patients' HRQOL as much as language or motor deficits caused by lesions in the left hemisphere. In left-dominant individuals, the right cerebral hemisphere contains important functions for spatial orientation and exploration. Lesions of the right

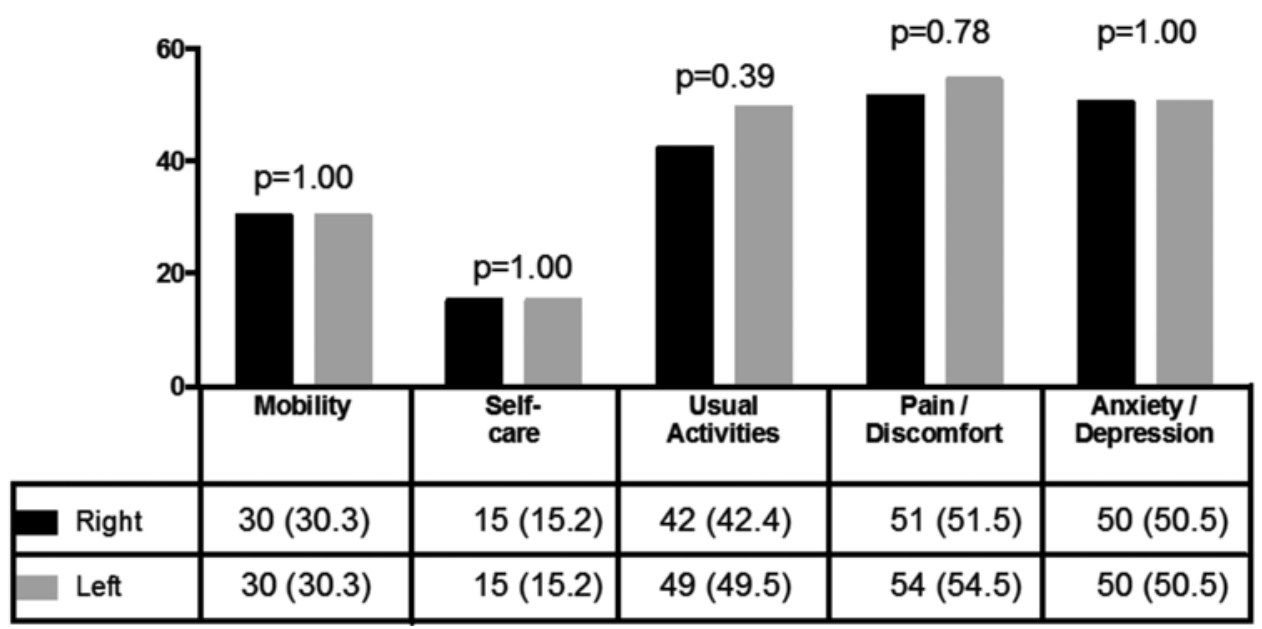

FIG. 3. Percentage of respondents in matched cohorts reporting any problem on the $E Q-5 D-3 L$ questionnaire. 
TABLE 2. Postoperative data of 215 patients with follow-up after 4-6 weeks*

\begin{tabular}{|c|c|c|c|}
\hline Variable & Rt-Sided Tumor & Lt-Sided Tumor & $p$ Value \\
\hline No. of patients & 98 & 117 & \\
\hline Females (\%) & $48(49)$ & $56(47.9)$ & 0.979 \\
\hline Mean age (SD) & $55.8(14.0)$ & $56.0(15.2)$ & 0.862 \\
\hline ASA class $(\%)$ & & & 0.760 \\
\hline $1-2$ & $78(79.6)$ & $90(76.9)$ & \\
\hline $3-4$ & $20(20.4)$ & $27(23.1)$ & \\
\hline Preop KPS score $(\%) \dagger$ & & & 0.637 \\
\hline 100 & $21(21.4)$ & $23(19.7)$ & \\
\hline 90 & $35(35.7)$ & $43(36.8)$ & \\
\hline 80 & $22(22.4)$ & $33(28.3)$ & \\
\hline 70 & $11(11.2)$ & $7(6)$ & \\
\hline$\leq 60$ & $9(9.2)$ & $11(9.4)$ & \\
\hline Histopathology (\%)‡ & & & 0.223 \\
\hline High-grade glioma & $38(38.8)$ & $54(46.2)$ & \\
\hline Low-grade glioma & $23(23.5)$ & $17(14.5)$ & \\
\hline Meningioma & $37(37.8)$ & $46(39.3)$ & \\
\hline Median preop tumor vol in $\mathrm{cm}^{3}$ (range) & $24.2(1.3-159.0)$ & $18.7(0.7-160.4)$ & 0.018 \\
\hline Median postop tumor vol in $\mathrm{cm}^{3}$ (range) & $0(0-113.7)$ & $0(0-160.4)$ & 0.531 \\
\hline Resection grade $(\%) \S$ & & & 0.583 \\
\hline Complete $(100 \%)$ & $50(52.6)$ & $64(55.2)$ & \\
\hline Subtotal $(\leq 99 \%)$ & $41(43.2)$ & $44(37.9)$ & \\
\hline Biopsy only & $4(4.2)$ & $8(6.9)$ & \\
\hline Postop KPS score (\%)ף & & & 0.354 \\
\hline 100 & $16(16.3)$ & $11(9.4)$ & \\
\hline 90 & $18(18.4)$ & $30(25.6)$ & \\
\hline 80 & $15(15.3)$ & $21(17.9)$ & \\
\hline 70 & $36(36.7)$ & $36(30.8)$ & \\
\hline$\leq 60$ & $13(13.3)$ & $19(16.2)$ & \\
\hline Mean change in KPS score (SD) ${ }^{* *}$ & $6(14)$ & $7(14)$ & 0.685 \\
\hline Median postop EQ-5D-3L index (range) & $0.80(-0.17$ to 1.0$)$ & $0.80(-0.59$ to 1.0$)$ & 0.957 \\
\hline Change in EQ-5D-3L index $\geq$ MCID $0.15(\%)$ & & & 0.793 \\
\hline Improved & $31(31.6)$ & $37(31.6)$ & \\
\hline Unchanged & $46(46.9)$ & $59(50.4)$ & \\
\hline Worse & $21(21.4)$ & $21(17.9)$ & \\
\hline
\end{tabular}

MCID = minimum clinically important difference.

* Boldface type indicates statistical significance.

$\dagger$ KPS scored by neurosurgeon on admission.

$\ddagger$ Confirmed by pathologist.

$\S$ Assessed from pre- and postoperative MR images.

ๆ KPS, scored by research nurse in structured phone interview at 1 month.

** Preoperative score - postoperative score.

hemisphere more often cause neglect, as initially found in up to $85 \%$ of right hemisphere strokes..$^{1,2,21}$ Right-sided lesions are also associated with anosognosia and various neuropsychiatric syndromes. ${ }^{4}$ These and other neurocognitive symptoms may profoundly hamper the patient's independence, social well-being, and many aspects of daily life, but may be less likely to be acknowledged by clinicians than, for example, dysphasia. In a previous study, we found very low (0.07) sensitivity values for neurosurgeons' assessment of cognitive deficits at hospital discharge, compared with the patients' own reports 1 month postoperatively ${ }^{5}$ We interpreted this as being due to the difficulties in detecting subtle, yet potentially disabling, cognitive impairment during a routine neurosurgical examination at hospital discharge. The same may possibly apply to neuropsychological/cognitive symptoms from right-hemisphere pathology. The often more diffuse character of right cerebral hemisphere symptoms may also lead to diagnosis at a later stage and thus may explain the larger tumor sizes at baseline in our patient population. One could therefore hypothesize that, to be clinically important enough to be diagnosed, a larger lesion is needed on the right side than on the left side. However, matching for tumor volume at baseline did not alter our findings when it comes to HRQOL. Based on our findings, it is tempting to conclude that tumor laterality per se is not a very important factor to consider when it comes to overall QOL, at least not at the group level. 
In the longitudinal analysis of 215 patients, clinical baseline data were again statistically evenly distributed except for preoperative tumor volume. Postoperative EQ$5 \mathrm{D}-3 \mathrm{~L}$ values and the change from pre- to postoperative EQ-5D-3L values were not statistically or clinically different in patients with right-sided versus left-sided lesions. Notably, we found no statistically significant laterality-dependent difference when looking at resection grades, indicating that our surgeons did not choose a more conservative strategy in patients with left-sided lesions.

The strengths of our study lie in the population-based referral, minimizing selection bias. Lack of informed consent to participate in the study was mainly due to administrative reasons/staffing, and we do not think that this affects the external validity of our results. Pseudorandomization "by nature" into right- and left-sided brain lesions provided well-matched cohorts already at baseline. Additionally, our matching procedure reduced the potential impact of tumor volume as a confounder. Furthermore, we included considerably more patients than the 63 per group we had aimed for to reach a power of about $80 \%$, and also the number of patients in the matched cohorts was well within the intended power. One weakness is that we did not register the actual handedness of our patients. Nevertheless, the vast majority of individuals have the dominant hemisphere located on the left side of the brain. ${ }^{3,12,14,20}$ Although we cannot exclude that use of other HRQOL instruments could have yielded different results, the EQ-5D-3L index is a validated and widely used tool that has clear practical advantages for HRQOL assessment in our patient group. It contains a simple scale, with only 3 possible response alternatives. In our experience, this makes it more likely for patients with slight cognitive impairment, concentration difficulties, or fatigue to actually complete the questionnaire. Additionally, the distinction between 3 possible response alternatives is manageable in a telephone interview at our chosen follow-up time at 4-6 weeks. In our study, the choice of follow-up at 4-6 weeks is based on the idea to allow a certain period of recovery after surgery, when adverse effects from chemotherapy or radiation therapy or symptoms of disease progression are still at a low level. It is also worth mentioning that there certainly exists a ceiling effect for the preoperative EQ5D-3L index values, influencing the calculated difference in EQ-5D-3L. A previous study found that the responsiveness of the EQ-5D-3L in this patient group is poor with respect to improvement, but the responsiveness with respect to deterioration is good ${ }^{18}$ Overall, this may lead to a seemingly low number of patients in our study population who improved after surgery.

In later years, modern imaging and anesthetic/surgical techniques such as awake surgery and intraoperative mapping techniques have facilitated safer removal of brain tumors even in eloquent areas, typically within the left hemisphere. Although eloquent functions, especially speech, are at greater risk when the left hemisphere is affected, our results imply that the patient-perceived difference in importance between the 2 cerebral hemispheres might not at all be as large. Despite resulting in qualitatively different symptoms, right-sided tumors may produce a symptom burden comparable to left-sided tumors, reflected by over- all patient-reported QOL. Clinical decision making should probably not primarily be based on laterality. Our findings could also be of relevance for clinical decisions in conditions other than brain tumors, such as stroke or traumatic brain injury.

\section{Conclusions}

We found no difference in overall HRQOL in patients with right-sided versus those with left-sided intracranial tumors, measured with the generic instrument EQ-5D-3L. This does not mean that location of a tumor is unimportant, but our findings challenge the traditional view of the left (dominant) cerebral hemisphere as the more important one for patients. Laterality alone should probably not be given too much weight in clinical decision making.

\section{Acknowledgments}

We would like to thank Linda M. Nordtvedt for assisting in data collection, Even H. Fyllingen for participating in assessment of medical images, and Øyvind Salvesen for valuable statistical advice.

\section{References}

1. Azouvi P, Samuel C, Louis-Dreyfus A, Bernati T, Bartolomeo P, Beis JM, et al: Sensitivity of clinical and behavioural tests of spatial neglect after right hemisphere stroke. J Neurol Neurosurg Psychiatry 73:160-166, 2002

2. Bowen A, McKenna K, Tallis RC: Reasons for variability in the reported rate of occurrence of unilateral spatial neglect after stroke. Stroke 30:1196-1202, 1999

3. Coren S: The Left-Hander Syndrome: the Causes and Consequences of Left-Handedness, ed 1. New York: Vintage Books, 1993

4. Cummings JL: Neuropsychiatric manifestations of right hemisphere lesions. Brain Lang 57:22-37, 1997

5. Drewes C, Sagberg LM, Jakola AS, Gulati S, Solheim O: Morbidity after intracranial tumor surgery: sensitivity and specificity of retrospective review of medical records compared with patient-reported outcomes at 30 days. J Neurosurg 123:972-977, 2015

6. Fink JN, Frampton CM, Lyden P, Lees KR: Does hemispheric lateralization influence functional and cardiovascular outcomes after stroke?: an analysis of placebo-treated patients from prospective acute stroke trials. Stroke 39:3335-3340, 2008

7. Golsari A, Cheng B, Sobesky J, Schellinger PD, Fiehler J, Gerloff C, et al: Stroke lesion volumes and outcome are not different in hemispheric stroke side treated with intravenous thrombolysis based on magnetic resonance imaging criteria. Stroke 46:1004-1008, 2015

8. Guyatt GH, Osoba D, Wu AW, Wyrwich KW, Norman GR: Methods to explain the clinical significance of health status measures. Mayo Clin Proc 77:371-383, 2002

9. Hahn CA, Dunn RH, Logue PE, King JH, Edwards CL, Halperin EC: Prospective study of neuropsychologic testing and quality-of-life assessment of adults with primary malignant brain tumors. Int J Radiat Oncol Biol Phys 55:992-999, 2003

10. Liu R, Solheim K, Polley MY, Lamborn KR, Page M, Fedoroff A, et al: Quality of life in low-grade glioma patients receiving temozolomide. Neuro Oncol 11:59-68, 2009

11. Mainio A, Hakko H, Niemelä A, Tuurinkoski T, Koivukangas J, Räsänen P: The effect of brain tumour laterality on anxiety levels among neurosurgical patients. J Neurol Neurosurg Psychiatry 74:1278-1282, 2003 
12. Mazoyer B, Zago L, Jobard G, Crivello F, Joliot M, Perchey $\mathrm{G}$, et al: Gaussian mixture modeling of hemispheric lateralization for language in a large sample of healthy individuals balanced for handedness. PLoS One 9:e101165, 2014

13. Nord E: EuroQol: health-related quality of life measurement. Valuations of health states by the general public in Norway. Health Policy 18:25-36, 1991

14. Peters M, Reimers S, Manning JT: Hand preference for writing and associations with selected demographic and behavioral variables in 255,100 subjects: the BBC internet study. Brain Cogn 62:177-189, 2006

15. Pickard AS, Neary MP, Cella D: Estimation of minimally important differences in EQ-5D utility and VAS scores in cancer. Health Qual Life Outcomes 5:70, 2007

16. Polin RS, Marko NF, Ammerman MD, Shaffrey ME, Huang W, Anderson FA Jr, et al: Functional outcomes and survival in patients with high-grade gliomas in dominant and nondominant hemispheres. J Neurosurg 102:276-283, 2005

17. Rogers MP, Orav J, Black PM: The use of a simple Likert scale to measure quality of life in brain tumor patients. J Neurooncol 55:121-131, 2001

18. Sagberg LM, Jakola AS, Solheim O: Quality of life assessed with EQ-5D in patients undergoing glioma surgery: what is the responsiveness and minimal clinically important difference? Qual Life Res 23:1427-1434, 2014

19. Salo J, Niemelä A, Joukamaa M, Koivukangas J: Effect of brain tumour laterality on patients' perceived quality of life. J Neurol Neurosurg Psychiatry 72:373-377, 2002

20. Seidman MD, Siegel B, Shah P, Bowyer SM: Hemispheric dominance and cell phone use. JAMA Otolaryngol Head Neck Surg 139:466-470, 2013

21. Stone SP, Halligan PW, Greenwood RJ: The incidence of neglect phenomena and related disorders in patients with an acute right or left hemisphere stroke. Age Ageing 22:46-52, 1993

22. Stummer W, Pichlmeier U, Meinel T, Wiestler OD, Zanella F, Reulen HJ: Fluorescence-guided surgery with 5-aminolevulinic acid for resection of malignant glioma: a randomised controlled multicentre phase III trial. Lancet Oncol 7:392401, 2006

23. Sundseth J, Sundseth A, Thommessen B, Johnsen LG, Altmann M, Sorteberg W, et al: Long-term outcome and quality of life after craniectomy in speech-dominant swollen middle cerebral artery infarction. Neurocrit Care 22:6-14, 2015

24. Trojanowski T, Peszyński J, Turowski K, Markiewicz P, Gościński I, Bielawski A, et al: Quality of survival of patients with brain gliomas treated with postoperative CCNU and radiation therapy. J Neurosurg 70:18-23, 1989

\section{Disclosures}

The authors report no conflict of interest concerning the materials or methods used in this study or the findings specified in this paper.

\section{Author Contributions}

Conception and design: all authors. Acquisition of data: all authors. Analysis and interpretation of data: Drewes, Jakola, Solheim. Drafting the article: all authors. Critically revising the article: all authors. Approved the final version of the manuscript on behalf of all authors: Drewes. Statistical analysis: Drewes. Study supervision: Solheim.

\section{Supplemental Information}

\section{Current Affiliation}

Dr. Drewes: Department of Anesthesiology, St. Olavs University Hospital; and Department of Neuroscience, Norwegian University of Science and Technology, Trondheim, Norway.

\section{Correspondence}

Christina Drewes, Department of Anesthesiology, St. Olavs University Hospital, Olav Kyrres Gate, Trondheim N-7005, Norway.email: christina.drewes@stolav.no. 